\title{
The prognosis of amalgam restorations depends on the alloy composition.
}

\author{
Letzel H, Van 'THof'MA, Marshall GW, Marshall SJ. The influence of the amalgam alloy on the survival ofamalgam \\ Restorations: a secondary analysis of multiple controlled clinical trials. I Dent Res. 1997; 76: 1787-98.
}

Objective To assess the influence of alloy composition on the survival of amalgam restorations.

Study selection Data from 14 independent controlled clinical trials on the oral behaviour of Classes I and 2 amalgam restorations, with a follow-up between five and 15 years.

Data extraction and synthesis All data from the 14 trials were cumulated and re-evaluated by secondary analysis. For the analysis, 3119 restorations were available, which were made from 24 different alloys by a group of seven operators. The alloys were divided into four groups according to their zinc content (zinccontaining and zinc-free) and their copper content (conventional and high-copper). During the follow-up of the trials, the restorations were annually assessed for failures, which were classified as to (1) restoration, (2) restorative process and (3) patient-related reasons. With the restoration-related failures, survival functions of the restorations were estimated by alloy and alloy group.

Results The total number of failed restorations was 481 , of which $77 \%$ were restoration-related and $14 \%$ process-related. Eighty percent of the restoration-related failures were due to some form of fracture of the amalgam. Restorations of conventional zinc-free alloys had the shortest survival. After 13 years only $25 \%$ survived. Zinc and a high copper content had an equally favourable influence on the survival rate, which was $70 \%$ after 13 years when either was present. The highest survival rates were of restorations of zinc-containing high-copper alloys: $85 \%$ after 13 years.

Conclusions This analysis suggests that the zinc and copper contents of the alloy influences survival of amalgam restorations.

Address for reprints: Dr GW Marshall, Department of Restorative Dentistry, Box 0758, University of California, San Fancisco, California 94143-0758, USA

\section{Commentary}

This paper describes a secondary analysis of fourteen controlled trials carried out between 1974 and 1988-89. A characteristic of these clinical trials is a high scientific internal validity due to strict control of all potentially confounding factors. There are no indications of selective patient dropout. All patients who entered the trials received at least six restorations, which reduced the bias introduced by a possible selective dropout. The statistics used in the analyses, take into account patient dropout by using censored survival time, which reduce the risk of making wrong inferences from the observation material. There is little reason to suspect that the authors' conclusion of an influence of the amalgam alloy on survival in this study is just a result of statistical modelling, but supports previous data from Osborne et al. ${ }^{1}$

The low external validity of the trials due to what the authors characterised as 'a narrowly selective sample' allowed the authors to assess an association between alloy composition and fractures. The patients consisted mainly of dental students and hospital staff and dentists, with practically non-existent secondary caries. The main reasons for restoration failure were marginal and bulk fractures of the material. However, in 'real-life' dentistry the main reason for restoration replacement is secondary caries $^{2,3}$ although it can be pointed out that a large part of such replacements may be due to poor differential-diagnosis. 1,2

The association between the selective study population and the failure modes in the trials may also explain the diverging opinions on the association between survival and alloy composition. ${ }^{3}$ A longitudinal study reported no association between amalgam alloy and survival. 4 Another study reported equal replacement rates of one conventional and four high-copper amalgams over 10 years. ${ }^{3}$ This study, however, identifies the patient caries activity and operator as more important predictors of restoration failure than the alloy composition. In Letzel et al, patient caries activity was almost absent, which otherwise influences restoration survival. In addition, table 6 displays a stronger relationship between survival and operator than to alloy if exluding the data for the two lowcopper-no-zinc alloys. However, the presentation of the findings only focusses on the effect of the alloy, while the operator effect is almost ignored.

Although many countries have introduced restrictions on using gamma-2 containing amalgams, these are still available. Dentists should carefully consider their rationale for continuing to use such alloys in view of the clinical benefit of using high-copper-non-zinc amalgam alloys.

1 Osborne IW, Summitt / B. The survival of amalgam restorations. I Dent Res. 1998; 77: 340 .

2 Quality evaluations of dental materials. Anusavice K J (ed). Quintessence Publishing Co, Chicago, 1989.

3 Jokstad A. Class 2 Cavity Preparations and Restoration Performance. Thesis. University of Oslo: 1992.

4 Kreulen C M et al. Replacement risk of amalgam treatment modalities: 15 -year results. J Dent 1998; 26: 627-32.

Asbjorn Jokstad

Associate Professor, University of Oslo, Norway 